La FMH soutient la campagne de prévention contre la grippe Cette année aussi, la FMH se rallie à la campagne de prévention contre la grippe lancée par le Collège des médecins de premier recours (CMPR). Cette nouvelle campagne, soutenue par l'Office fédéral de la santé publique (OFSP), vise à réduire morbidité et mortalité, diminuer le risque de contagion et de transmission du virus et endiguer l'augmentation des coûts dans le secteur de la santé.
La FMH invite donc le corps médical - de même que l'ensemble du personnel médical, soignant et paramédical, ainsi que nos confrères dentistes et pharmaciens - à largement s'associer à cette campagne et à soutenir les efforts des organisations des médecins de premier recours, qui organisent le 4 novembre 2005 leur seconde journée nationale de vaccination.

Ressort santé et prévention de la FMH

\title{
Quelle information faut-il donner lors de la vaccination dans le cadre de la Journée nationale de vaccination contre la grippe?*
}

\author{
Renseignements sur la situation juridique et offre d'une solution pratique
}

\section{U. Grüninger}

Les médecins de premier recours vaccinent un grand nombre de leurs patients contre la grippe. La question du contenu et de la manière dont un patient doit être renseigné au sujet de la vaccination contre la grippe n'est donc pas nouvelle, mais elle a été réactualisée par la Journée nationale de vaccination contre la grippe. L'analyse juridique des problèmes et conséquences d'une complication sévère - encore que bien improbable - pourrait aussi décourager les médecins engagés; mais par bonheur, la solution n'est pas aussi compliquée que le problème.

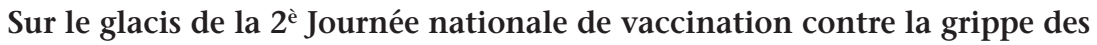
médecins de premier recours suisses, qui aura lieu le 4 novembre 2005, le présent article résume les exigences légales d'information des patients avant une vaccination contre la grippe et offre aux collègues intéressés une solution pragmatique et juridiquement fondée.

Deutsch erschienen

in Nr. 37
La solution ne doit pas être aussi compliquée que le problème.

Steve de Shazer [1]

\footnotetext{
* La version originale de cet article a paru d'abord dans PrimaryCare 2005;5(34/35):689-91.
}

Correspondance:

Dr Ueli Grüninger

Collège de médecine

de premier recours (CMPR)

Landhausweg 26

CH-3007 Berne

E-mail: ueli.grueninger@hin.ch née peuvent se faire vacciner contre la grippe dans un cabinet médical prenant part à l'action, sans s'annoncer au préalable et contre paiement d'une participation forfaitaire. En règle générale, la vaccination est pratiquée par le personnel du cabinet médical, séparément de la consultation médicale.

\section{Situation du problème du point de vue juridique}

La vaccination contre la grippe constitue une vaccination susceptible d'effets indésirables mais aussi une mesure efficace contre cette maladie, où la vraisemblance d'effets secondaires sévères est nettement plus petite que le risque de complications sérieuses d'une grippe.

Pourtant, quelques collègues nous ont fait part [3] de leur souci que du point de vue juridique, les exigences d'information de la personne à vacciner, par exemple de la part de l'OFSP [4], soient exagérées, en particulier dans le cadre d'une telle Journée de vaccination et que ces exigences seraient par conséquent impraticables, de sorte qu'un médecin courrait le danger de se voir exposé à des poursuites judiciaires pour information insuffisante en cas de complication sévère et pourrait même encourir un grave préjudice financier en vertu de l'engagement de sa responsabilité civile. Un collègue a allégué que l’Etat se 
déchargeait du risque juridique (responsabilité civile en cas de complications) sur les médecins de premier recours, quand bien même la vaccination contre la grippe représente un intérêt fondamental majeur pour la santé de la population.

\section{Procédé de clarification juridique}

Nous avons soumis ces questions au service juridique de la FMH et de l'OFSP, en priant ces instances de clarifier la situation juridique et de formuler les points dont il faut tenir compte pour une vaccination dans le cadre de la Journée de vaccination. Sur la base de cette clarification, nous avons, en collaboration avec ces juristes et les collègues praticiens, voulu définir pratiquement comment un titulaire de cabinet médical participant à la Journée de vaccination peut se conformer suffisamment aux prescriptions légales tout en respectant le principe de proportionnalité.

\section{Situation du point de vue juridique [5]}

Fondamentalement et formellement, la situation juridique d'une vaccination contre la grippe dans le cadre d'une Journée de vaccination n'est pas différente de celle de vaccinations dans d'autres circonstances, donc dans le cadre de la routine d'une consultation normale ou comme généralement dans l'activité professionnelle du médecin: le médecin exécute un mandat que lui a confié le patient et si le médecin le fait selon les règles usuelles de l'art et qu'il a aussi documenté l'information donnée au patient, il satisfait à son devoir et n'a donc pas à craindre d'une quelconque poursuite judiciaire au cas où une complication devait apparaître. Deux choses sont donc à considérer: l'information fait partie du devoir professionnel et en cas de doute, l'information donnée doit pouvoir être démontrée.

Comme toutes les vaccinations non obligatoires en vertu d'une disposition légale, la vaccination contre la grippe intervient dans le cadre d'un mandat de droit privé donné au médecin par le patient.

En cas de comportement erroné, c'est la responsabilité du médecin qui est engagée en première ligne. Dans le cas d'un dommage consécutif à une vaccination ordonnée ou recommandée par les autorités, la responsabilité du canton est subsidiairement engagée. Un devoir d'indemnisation de la part du canton n'entre donc en ligne de compte que lorsqu'on ne peut pas obtenir de couverture suffisante de la part de l'intervenant primaire (responsabilité civile professionnelle du médecin, assurances sociales). Le canton ne verse alors d'indemnité que pour le solde restant après mise à contribution des autres assureurs en responsabilité civile (couverture de la différence).

Pour des raisons de santé publique, l'OFSP a certes un intérêt à ce que la vaccination contre la grippe soit pratiquée, mais on ne peut pas se substituer aux médecins pour leur responsabilité d'une information correcte des patients et de l'exécution de la vaccination dans les règles de l'art. Cependant, dans le sens d'une prestation d'aide aux médecins vaccinant, on met à disposition un moyen d'information de l'Etat d'une haute qualité (brochure à l'intention du personnel médical, brochures d'information pour les patients).

Du point de vue juridique, l'information exigée est toujours mesurée en fonction des caractéristiques du cas particulier, de sorte qu'on ne peut pas dire globalement quels éléments doivent concrètement faire partie de l'entretien d'information. Dans le cadre de la Journée de vaccination contre la grippe, une information adéquate devrait en règle générale couvrir au moins les domaines suivants: questions sur l'état de santé général du patient (en relation avec la vaccination), renseignements sur la mesure envisagée (vaccination contre la grippe) et les risques qui lui sont liés (effets indésirables possibles, etc.), ainsi que sur les coûts et le mode de paiement. Dans les directives du Supplementum XVI de l'OFSP, il s'agit de recommandations générales aux médecins qui pratiquent des vaccinations; on peut tenir compte des particularités d'une vaccination dans le cadre de la Journée de vaccination contre la grippe.

\section{L'information et la documentation en pratique}

Pour l'interprétation adéquate et la pratique conforme, deux aspects sont importants:

- l'échange d'information sur la vaccination contre la grippe avec le candidat à la vaccination (contre-indications, information sur la protection attendue et les possibles effets secondaires, paiement);

- la documentation de l'information donnée et de l'évaluation des risques.

Cette information et cette documentation peuvent très bien être faites, comme la vaccination elle-même, par du personnel non médical, à condition que ce personnel ait été instruit en conséquence par le titulaire du cabinet. Ce qui compte, c'est que tout le processus soit exécuté adéquatement et aussi documenté. 
En d'autres termes: une vaccination dans le cadre de la Journée de vaccination contre la grippe n'est pas différente d'une autre activité médicale au cabinet. Le médecin titulaire du cabinet est responsable de ce qui se passe dans son cabinet et a intérêt à ce que lui et ses collaboratrices et collaborateurs pratiquent l'information et l'exécution dans les règles de l'art et le plus grand soin.

Qui ne le fait pas encourt le risque qu'un patient présentant une complication sérieuse certes statistiquement très rare dans le cas de la vaccination contre la grippe - introduise une action en responsabilité civile.

Comment le titulaire du cabinet veut concrètement assumer son devoir de prudence tient à son propre jugement.

\section{Une feuille de documentation}

Pour le cas de la Journée de vaccination contre la grippe et spécialement pour les vaccinations de personnes qui ne font pas partie des patients connus du médecin vaccinant, le CMPR offre aux collègues intéressés une aide pratique sous la forme d'une feuille de documentation (une page A4), qui correspond aux exigences d'information décrites plus haut (fig. 1).

Le CMPR a développé cette «Feuille de documentation Journée de vaccination contre la grippe» en collaboration avec la FMH et l'OFSP, et en complément à la brochure de l'OFSP destinée aux patients. Sur cette feuille sont énoncés les quatre principaux domaines faisant, pour des raisons médicales et légales, partie de l'information avant une vaccination contre la grippe: recensement des contre-indications, information reçue au moyen de la brochure de l'OFSP, consentement du patient et vaccin utilisé [6].

Au cabinet médical, le déroulement peut être le suivant: lorsqu'une personne manifeste son intérêt à être vaccinée, on lui donne d'abord à lire la brochure de l'OFSP; ensuite la personne qui vaccine (en règle générale l'assistante médicale lors de la Journée de vaccination contre la grippe) utilise la feuille de documentation d'une part comme check-liste pour l'information et le recensement des contre-indications et d'autre part pour documenter l'entretien pré-vaccination (cinq cases à cocher, visa du cabinet).

Cette feuille de documentation pourra être téléchargée à partir du site Internet du Collège de médecine de premier recours (www. kollegium.ch) ainsi qu'à partir du site Internet grippe de l'OFSP (www.grippe.admin.ch). La feuille de documentation fait partie des moyens auxiliaires offerts pour la Journée de vaccination contre la grippe et son utilisation est facultative, comme déjà mentionné plus haut.

\section{Remarque finale: Tempora mutantur ou sur l'arête effilée entre proportionnalité et esprit du temps}

Bon nombre parmi nous, médecins de premier recours interrogés, ont considéré comme infondées ou exagérées les arrière-pensées juridiques de quelques collègues et malheureusement insisté en prétendant que de telles interpellations pourrait ouvrir une boîte de Pandore juridique. Mais ces soucis sont au contraire compréhensibles sur la toile de fond d'une gestion du cabinet centrée sur le médecin et la question de la proportionnalité se pose en fait bel et bien.

D'autre part, les temps ont changé et avec eux les exigences de la société quant à notre devoir de rendre des comptes. Cela a pour conséquence et on le voit justement ici - que ceux qui se sont exprimés dans le courrier des lecteurs perçoivent une plainte en responsabilité civile et même une condamnation comme une réelle possibilité et un nouveau type de menace.

Ce dilemme est bien réel mais aussi pas nouveau, la Journée de vaccination contre la grippe n'a été qu'une occasion de le thématiser. Vouloir exorciser, nier ou fermer les yeux face à ce dilemme n'apporterait rien: nous devons accepter que dans notre travail de médecin, nous ayons à poser consciemment nos pas sur l'arête effilée entre proportionnalité pratique et minoration du risque juridique. Cela n'est pas forcément plus facile avec les yeux bien ouverts, mais certainement meilleur. Et comme je l'ai déjà dit: par bonheur, les médecins de premier recours sont habitués à vivre avec un certain degré d'incertitude.

Des informations sur la Journée nationale de vaccination contre la grippe par les médecins de premier recours du 4 novembre 2005 sont disponible auprès de l'adresse de correspondance et sur le site web du Collège de médecine de premier recours: www.kollegium.ch.

\section{Références}

1 de Shazer S. Wege der erfolgreichen Kurztherapie. Stuttgart: Klett; 1989.

2 Appartiennent à ces groupes à risque: individus âgés de plus de 65 ans, personnes atteintes de maladies chroniques, personnes séjournant dans des homes, personnel médical et personnel soignant ainsi que personnes de contact qui pourraient contaminer les personnes à risque (cf. PrimaryCare 2005;5[17]:388-9 ainsi que Bull Méd Suisses 2005;86[17]:1009). 
3 Lettres de lecteurs König (PrimaryCare 2005 5[10]:221) et Schiller (PrimaryCare 2005; 5[17]:392), ainsi que diverses autres lettres ainsi que commentaires dans les réponses au questionnaire sur la Journée de vaccination 2004.

4 «Recommandations générales pour les vaccinations», dans Supplementum XVI au classeur bleu des maladies infectieuses de l'OFSP, section Questions juridiques (paragraphes: consultation de vaccination, consentement, anamnèse et documentation).
5 L'auteur remercie l'avocat Thomas Bertschy du service juridique de l'OFSP et Hanspeter Kuhn du service juridique de la FMH pour leur précieuse collaboration à la clarification des exigences légales et formelles quant à l'information des patients.

6 Comme nous venons de l'apprendre après la fin de notre travail, la Société de médecine de Bâle MedGes a aussi développé un questionnaire (surtout pour la saisie des contre-indications) pour son action de vaccination contre la grippe 2005 (www.medges.ch).

Figure 1

Feuille de documentation Journée nationale de vaccination contre la grippe. Téléchargement de www.kollegium.ch ou www.grippe.admin.ch.
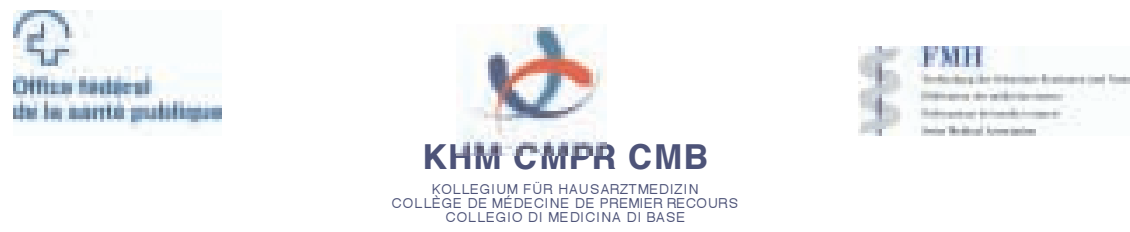

Formulaire pour la Journée de vaccination contre la grippe 2005

Nom :

Prénom :

Année de naissance :

Adresse : .

NP/Lieu :

\section{Contre-indications}

- Avez-vous actuellement de la fièvre ?

Non Oui

- Etes-vous hypersensible au blanc d'oeuf ?

$\square \quad \square$ report de la vaccination

- Problèmes rencontrés lors de vaccinations précédentes ?

$\square$ avis médical

$\square$ avis médical

\section{Information au patient sur la vaccination contre la grippe :}

La brochure pour patients de l'Office Fédéral de la Santé Publique (dépliant d'information sur la vaccination contre la grippe) a été distribuée et les points suivants ont été discutés:

- Contre quoi protège la vaccination contre la grippe ?

- Quels sont les éventuels effets secondaires ${ }^{1}$ et les risques ${ }^{2}$ de la vaccination contre la grippe?

- Qui devrait se faire vacciner?

- Qui paie?

\section{Décision du patient}

Accepte de se faire vacciner

Vaccin utilisé :

${ }^{1}$ Rougeurs autour de la zone d'injection, douleurs et démangeaisons ; plus rarement légère fièvre, nausées ou douleurs musculaires ; très rarement, tuméfaction, urticaire, asthme allergique ou réaction allergique immédiate.

${ }^{2}$ Le risque de graves complications suite à une grippe est plus important que celui d'effets secondaires sérieux secondaires à la vaccination. 